\title{
Utilidad de la ecografía clínica en el diagnóstico del paciente con disnea
}

\author{
Use of clinical echography in dyspnea diagnosis
}

\author{
Silvia Remesal Blanco, Alicia Fuente Gaforio, Beatriz Honrado Galán, María Mateos González, \\ Juan Torres Macho, Gonzalo García de Casasola Sánchez \\ Servicio de Medicina Interna-Urgencias. Hospital Universitario Infanta Cristina. Parla. Madrid
}

\section{Introducción}

La disnea es un síntoma frecuente. Tiene una amplia variedad de posibilidades etiológicas y no siempre resulta sencillo establecer un diagnóstico correcto, especialmente en personas ancianas, obesas o en pacientes con antecedentes de enfermedad pulmonar obstructiva crónica (EPOC).

La disnea puede deberse a procesos potencialmente graves en los que un diagnóstico precoz y el inicio del tratamiento médico adecuado son muy importantes ${ }^{1,2}$.

El desarrollo de equipos ecográficos portátiles y de gran calidad a un precio asequible ha permitido una enorme difusión de ecografía en múltiples especialidades (medicina interna, urgencias, cuidados intensivos, anestesia, reumatología, neumología, etc. ). La ecografía clínica, es decir, la realizada por el médico directamente responsable del paciente a pie de cama facilita el diagnóstico precoz del enfermo con disnea en múltiples ámbitos (urgencias, planta de hospitalización, unidades de cuidados intensivos y reanimación), incluido el medio extrahospitalario ${ }^{3}$.

\section{Etiología de la disnea}

En la tabla 1 mostramos las causas más frecuentes de disnea. En la mayoría de los casos la disnea está relacionada con patología respiratoria o cardiaca, aunque existen diagnósticos alternativos.

A pesar de una correcta valoración clínica inicial, en un porcentaje significativo de pacientes no se puede establecer con exactitud la etiología de la disnea y existen al menos dos o más posibilidades diagnósticas. De esta forma surge el concepto de "disnea indiferenciada" (tabla 2) ${ }^{2}$.

Un reto diagnóstico, por ejemplo, es la agudización del enfermo con EPOC que puede ser debida a múltiples circunstancias (infección respiratoria, embolia de pulmón, neumotórax, insuficiencia cardiaca, cáncer de pulmón).

\section{Evaluación diagnóstica del paciente con disnea}

La base del diagnóstico del enfermo con disnea se sustenta en la historia clínica y exploración física. Dependiendo de la valoración clínica inicial se solicitan, si es necesario, pruebas complementarias para esclarecer su etiología. Entre las pruebas iniciales básicas destaca ECG, gasometría, radiografía de tórax y analítica (hemograma, bioquímica elemental, dímero D, péptidos natriuréticos)

El diagnóstico de la insuficiencia cardiaca puede ser difícil, sobre todo en estadios iniciales, ya que muchos de sus síntomas y signos son inespecíficos. En estos casos, puede ser útil la determinación los péptidos natriuréticos. Probablemente la mejor utilidad de los mismos es que si sus niveles son nor-

Tabla 1. Causas más frecuentes de disnea

\begin{tabular}{|c|c|c|}
\hline $\begin{array}{l}\text { Disnea } \\
\text { de origen } \\
\text { respiratorio }\end{array}$ & $\begin{array}{c}\text { Disnea } \\
\text { de origen } \\
\text { cardiovascular }\end{array}$ & $\begin{array}{c}\text { Disnea con sistema } \\
\text { respiratorio y } \\
\text { cardiovascular } \\
\text { normales }\end{array}$ \\
\hline $\begin{array}{l}\text { Enfermedades } \\
\text { pulmonares obstructivas: } \\
\text { - EPOC } \\
\text { - Asma } \\
\text { Enfermedades de la caja } \\
\text { torácica: } \\
\text { - Cifoescoliosis } \\
\text { - Patología de los músculos } \\
\text { respiratorios (miastenia } \\
\text { gravis, sd de Guillain- } \\
\text { Barré) } \\
\text { Patología pleural: } \\
\text { - Derrame pleural de gran } \\
\text { cuantía } \\
\text { - Neumotórax } \\
\text { Enfermedades del } \\
\text { parénquima pulmonar: } \\
\text { - Enfermedades intersticiales } \\
\text { - Neumonía } \\
\text { - Cáncer de pulmón }\end{array}$ & $\begin{array}{l}\text { Cardiopatía izquierda: } \\
\text { - Disfunción sistólica } \\
\text { - Disfunción diastólica } \\
\text { - Valvulopatía mitral y/o } \\
\text { aórtica } \\
\text { Enfermedades del } \\
\text { pericardio: } \\
\text { - Taponamiento cardiaco } \\
\text { - Pericarditis constrictiva } \\
\text { Enfermedades } \\
\text { vasculares pulmonares: } \\
\text { - Embolia de pulmón } \\
\text { - Hipertensión pulmonar }\end{array}$ & $\begin{array}{l}\text { Anemia } \\
\text { Obesidad } \\
\text { Inadecuada preparación } \\
\text { física }\end{array}$ \\
\hline
\end{tabular}

Tabla 2. Causas más frecuentes de disnea de disnea de etiología incierta (o indiferenciada)

\begin{tabular}{|l|l|}
\hline Cardiopatía isquémica aguda & Derrame pleural \\
Insuficiencia cardiaca aguda & Neumonía \\
EPOC agudizado & Embolia de pulmón \\
Cáncer de pulmón & Asociación de patología pulmonar y \\
Edema pulmonar no cardiogénico & cardiaca de forma simultánea \\
\end{tabular}


males se puede excluir una patología cardiaca significativa como causa de la disnea sin necesidad de realizar un ecocardiograma. No obstante, es muy importante recordar que los niveles de péptidos natriuréticos pueden estar elevados, además de en la insuficiencia cardiaca, en el tromboembolismo pulmonar, en la insuficiencia renal y en individuos ancianos. Por tanto, la elevación de sus niveles no garantiza el diagnóstico de insuficiencia cardiaca ${ }^{4}$.

\section{Utilidad de la ecografía clínica en la valoración del paciente con disnea}

En la evaluación diagnóstica del paciente con disnea, la ecografía clínica sirve para complementar la exploración física tradicional y permite establecer el diagnóstico etiológico en un número significativo de enfermos, incluso antes de disponer de pruebas complementarias como la radiografía de tórax o las determinaciones analíticas. No obstante, es preciso recalcar que, sin lugar a dudas, la anamnesis y la exploración física tradicionales constituyen el eje de la orientación diagnóstica del paciente con disnea.

Para la valoración ecográfica completa del enfermo con disnea deberemos realizar ecografía pulmonar, ecocardiograma básico y ecografía por compresión simplificada de las extremidades inferiores. Con un adecuado entrenamiento esta valoración ecográfica puede realizarse en menos de 15 minutos ${ }^{2,5}$.

\section{1 Ecografía pulmonar}

La ecografía pulmonar permite detectar la presencia de edema intersticial, derrame pleural, condensaciones (neumonía) y neumotórax. Tiene una gran utilidad para el diagnóstico de la insuficiencia cardiaca izquierda.

En manos expertas la ecografía pulmonar tiene mayor precisión diagnóstica para la neumonía y el neumotórax que la radiografía de tórax ${ }^{6-8}$

\section{1.1 Técnica}

Para la evaluación de tejidos blandos, pared torácica y pleura, la sonda ideal será la lineal o de alta frecuencia (5-7 MHz). Es la sonda adecuada para el diagnóstico de neumotórax.

Para la exploración de patología pulmonar o para la inspección de áreas más profundas, emplearemos sondas convex o microconvex de 2'5 a $5 \mathrm{MHz}$.

La posición ideal del paciente será la de sentado o en semisedestación y se explorará de forma sistemática, siguiendo un orden y en las áreas en que habitualmente se coloca el fonendoscopio a cualquier enfermo para la auscultación convencional (Figura 1).

En la imagen ecográfica de pulmón normal (Figura 2) las sombras de ambas costillas lateralmente y la línea pleural entre ambas, conforman algo parecido a las alas de un murciélago y por ello se denomina "signo del murciélago". Con la sonda lineal se puede observar perfectamente el desplazamiento de las hojas pleurales en la línea pleural.
Figura 1. Exploración ecográfica del pulmón. La posición ideal del paciente será la de sentado o en semi-sedestación y se explorará de forma sistemática las áreas en las que habitualmente se coloca el fonendoscopio a cualquier enfermo para la auscultación convencional.

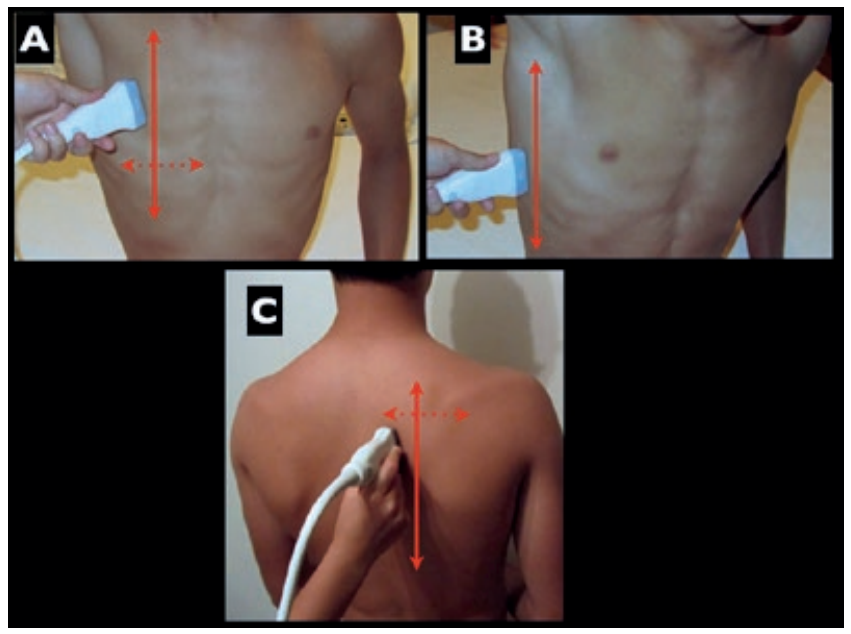

Figura 2. Imagen ecográfica de un pulmón normal. Se aprecian las sombras acústicas de las costillas y la línea pleural.

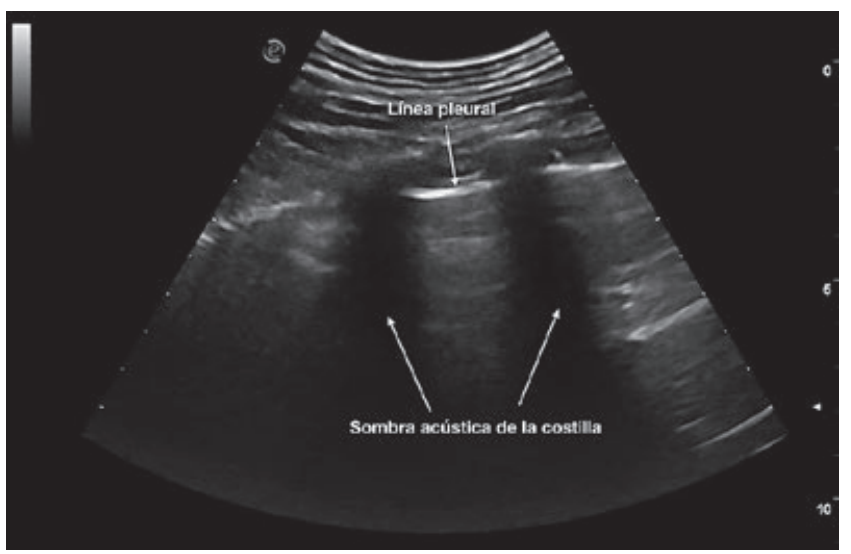

Figura 3. Líneas B. Se trata de líneas verticales como rayos láser (flechas) que se extienden hasta el fondo de la pantalla.

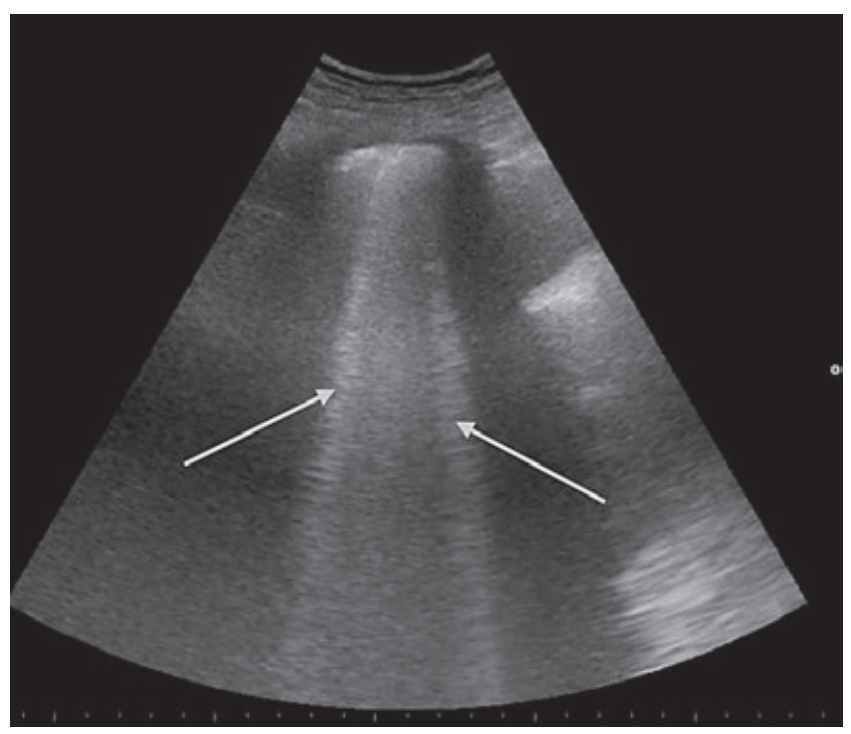




\section{1.2 Utilidad clínica}

\section{1. 2. 1 Patología intersticial}

En la patología intersticial (edema agudo de pulmón cardiogénico o no cardiogénico, fibrosis pulmonar) se aprecian las denominadas líneas B, que parten desde la línea pleural, son verticales como rayos láser que se extienden hasta el fondo de la pantalla sin palidecer y se mueven sincrónicamente con los movimientos respiratorios. La presencia de múltiples líneas $B$ en espacios pleurales antero-superiores del tórax, de forma bilateral y simétricas, en un contexto de insuficiencia respiratoria aguda sirve para establecer el diagnóstico de edema agudo de pulmón con muy alta probabilidad (Figura 3).

\section{1. 2. 2 Derrame pleural}

El derrame pleural se ve como una banda anecoica que separa las dos hojas pleurales (Figura 4). La ecografía es una técnica muy sensible (100\%) y específica $(99,7 \%)$ para la detección de derrame pleural y es insustituible como guía para la realización de la toracocentesis.

La presencia de derrame pleural bilateral nos obligar a descartar insuficiencia cardiaca como causa de la disnea.

Un derrame pleural unilateral debe hacer sospechar diagnósticos alternativos a la insuficiencia cardiaca. En este sentido es preciso tener en cuenta que en hasta un $50 \%$ de las neumonías se acompañan de derrame pleural. La presencia de septos y loculaciones en el derrame pleural (Figura 5) obliga a descartar un empiema y muy probablemente deba ser evacuado.

\section{1. 2. 3 Neumonía}

En algunos estudios se ha demostrado que la ecografía, en manos expertas, tiene una exactitud diagnóstica superior a la radiografía de tórax para el diagnóstico de neumonía ${ }^{6}$. La fiabilidad de la radiología de tórax en el diagnóstico de la neumonía es extremadamente variable, especialmente en pacientes deshidratados, inmunocomprometidos 0 ancianos. Además, en muchos enfermos es muy difícil realizar una radiografía de tórax de calidad.

Cuando se produce una ocupación de los alveolos por líquido inflamatorio, agua u otro material (condensación), el pulmón se convierte en una masa sólida capaz de transmitir los ultrasonidos y adquiere una ecogenicidad similar a la del parénquima hepático o esplénico. En su interior también es posible la visualización del broncograma aéreo (pequeñas imágenes puntiformes hiperecoicas en el interior de la condensación)(figura 5).

Ya hemos comentado que en un $50 \%$ de las neumonías es posible detectar derrame pleural. En una radiografía de tórax suele ser difícil precisar si una neumonía se acompaña de derrame pleural.

\section{1. 2. 4 Neumotórax}

El neumotórax es una entidad poco frecuente como causa de disnea. Sin embargo, su incidencia es relativamente
Figura 4. Derrame pleural en mujer joven con síndrome nefrótico. En la radiografía de tórax se aprecia pinzamiento de ambos senos costo-frénicos. En la ecografía pulmonar de la base pulmonar derecha se observa con nitidez el derrame pleural, el parénquima pulmonar y el hígado.

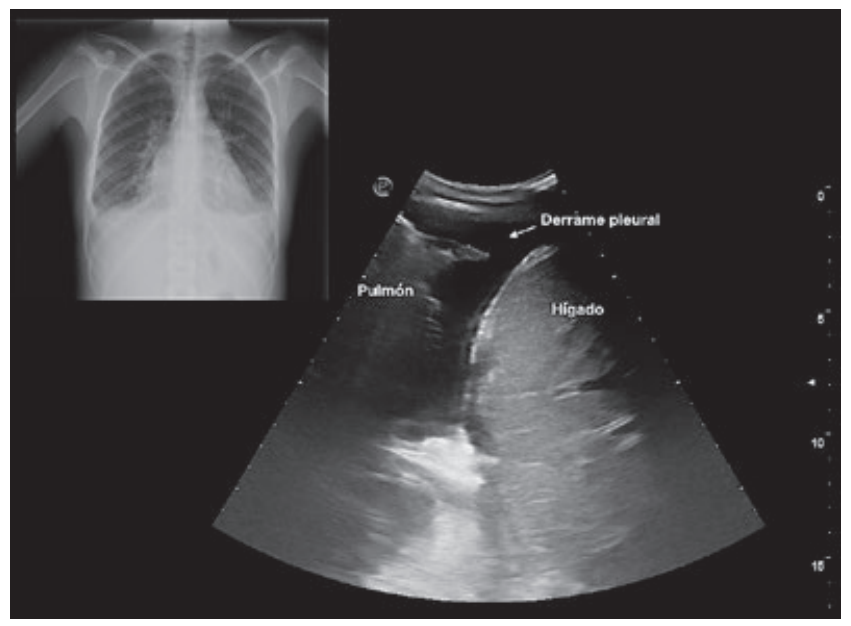

Figura 5. A) Ecografía pulmonar de paciente con neumonía y derrame pleural asociado (flechas). En la condensación neumónica se aprecia un pequeño punteado hiperecoico (blanco) que se corresponde con el broncograma aéreo B) Empiema. El derrame pleural está tabicado con múltiples septos en su interior.
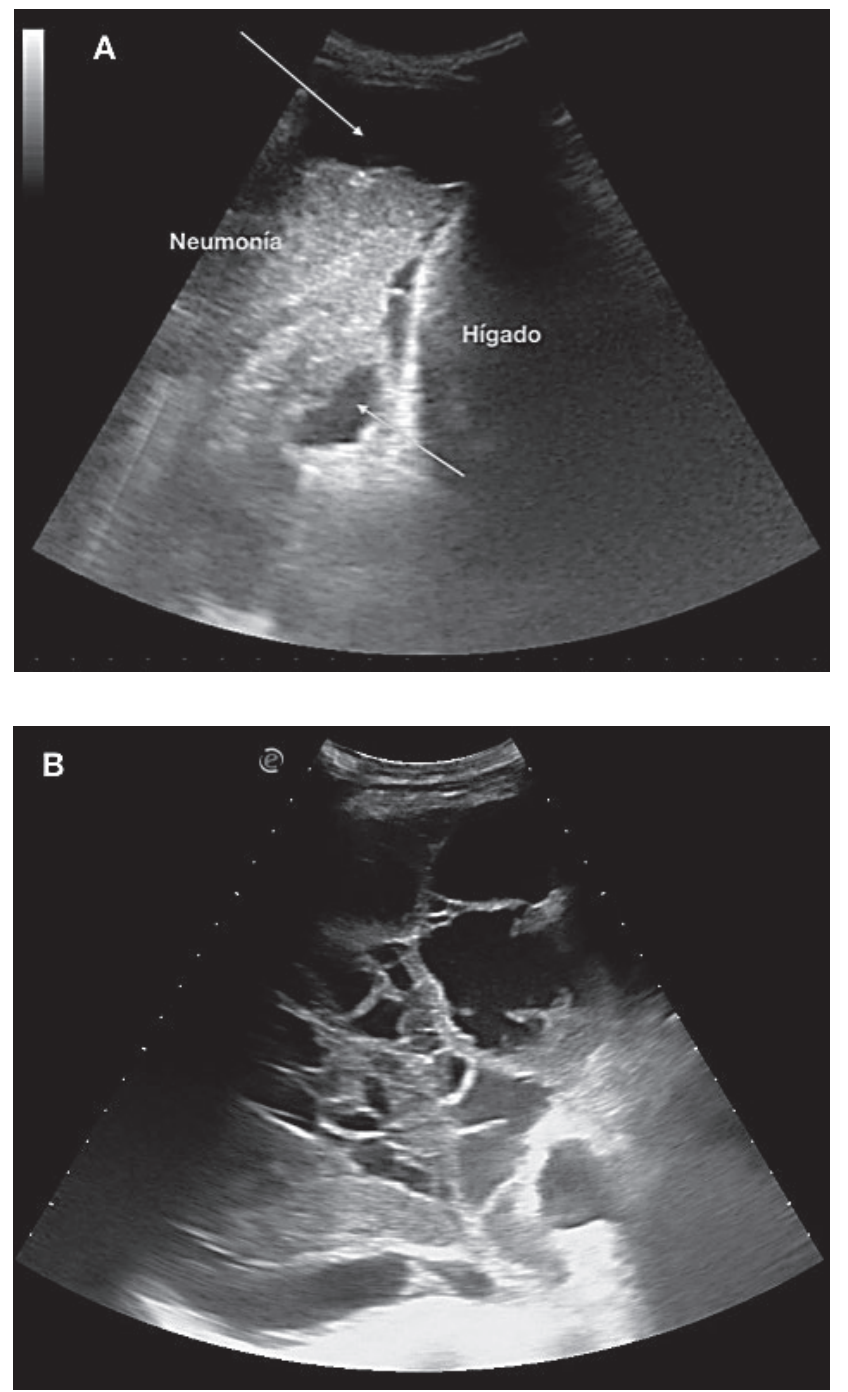
alta en enfermos con politraumatismos y en los sometidos a ventilación mecánica. La radiografía de tórax puede llegar a obviar hasta el 30-40\% de los neumotórax, sobretodo si son anteriores, en situaciones de urgencia y en enfermos con politraumatismos.

Desde el punto de vista ecográfico lo más característico del neumotórax es la desaparición del desplazamiento entre las hojas pleurales ("lung sliding") en la línea pleural. Como ya hemos comentado anteriormente la pleura se visualiza mucho mejor con la sonda lineal de alta frecuencia. La ausencia de "lung sliding" se detecta con mayor precisión con el modo M (figura 6).

La presencia de líneas B descarta el neumotórax en la zona explorada.

\section{2 Ecocardiografía clínica}

La ecocardiografía clínica realizada a pie de cama no sustituye al estudio reglado realizado por el cardiólogo. Se trata de una herramienta que añadida a la historia clínica (anamnesis y exploración física) y a pruebas complementarias básicas (ECG, radiografía de tórax) proporciona una mayor capacidad diagnóstica y puede agilizar y orientar el enfoque terapéutico inicial de los pacientes con disnea ${ }^{1,2,5,9}$

\section{2. 1 Técnica}

La sonda adecuada para la realización de un ecocardiograma es la sonda sectorial, de baja frecuencia (3-5 MHz), ya que su morfología facilita la transmisión de los ultrasonidos a través de los espacios intercostales.

En ecocardiografía clínica básica vamos a emplear la mayor parte del tiempo de exploración el modo 2D, aunque en ocasiones utilizaremos el modo M para determinadas mediciones y el Doppler-color para la detección de insuficiencias valvulares significativas. Para la visualización del corazón vamos a utilizar cuatro planos básicos: paraesternal eje largo y eje corto, apical cuatro cámaras y subxifoideo (figura 7).

\section{2. 2 Utilidad clínica}

\section{2. 2. 1 Valoración ecocardiográfica del paciente con sospecha de insuficiencia cardiaca}

El diagnóstico de la insuficiencia cardiaca, una de las causas más frecuentes de disnea, se basa en la historia y exploración física (síntomas y signos físicos). Sin embargo, para su diagnóstico de certeza es obligado constatar lesiones estructurales cardiacas, como son la disfunción sistólica y/0 diastólica ventricular izquierda o las valvulopatías significativas ${ }^{4}$.

Uno de los aspectos más útiles en ecocardiografía clínica es la estimación de la función sistólica del ventrículo izquierdo (FEVI). La valoración de la FEVI se puede realizar de forma cualitativa (evaluación visual subjetiva). Cuando se tiene cierta experiencia el grado de concordancia entre las diversas formas de cuantificar de forma objetiva la FEVI (ej: método Simpson) y la estimación visual de la misma es bastante elevado (Figura 8).
Figura 6. Neumotórax. La ausencia de desplazamiento entre las hojas pleurales se aprecia mejor con el modo M. En condiciones normales (A) el desplazamiento entre ambas hojas pleurales en modo M condiciona el signo de la orilla (por debajo de la línea pleural se aprecia un patrón granular parecido a la arena de la playa). La ausencia de desplazamiento entre las pleuras característico del neumotórax (B) ocasiona el signo de la orilla o de la estratosfera (por debajo de la línea pleural se aprecia un patrón lineal similar al que se observa por encima de la misma).
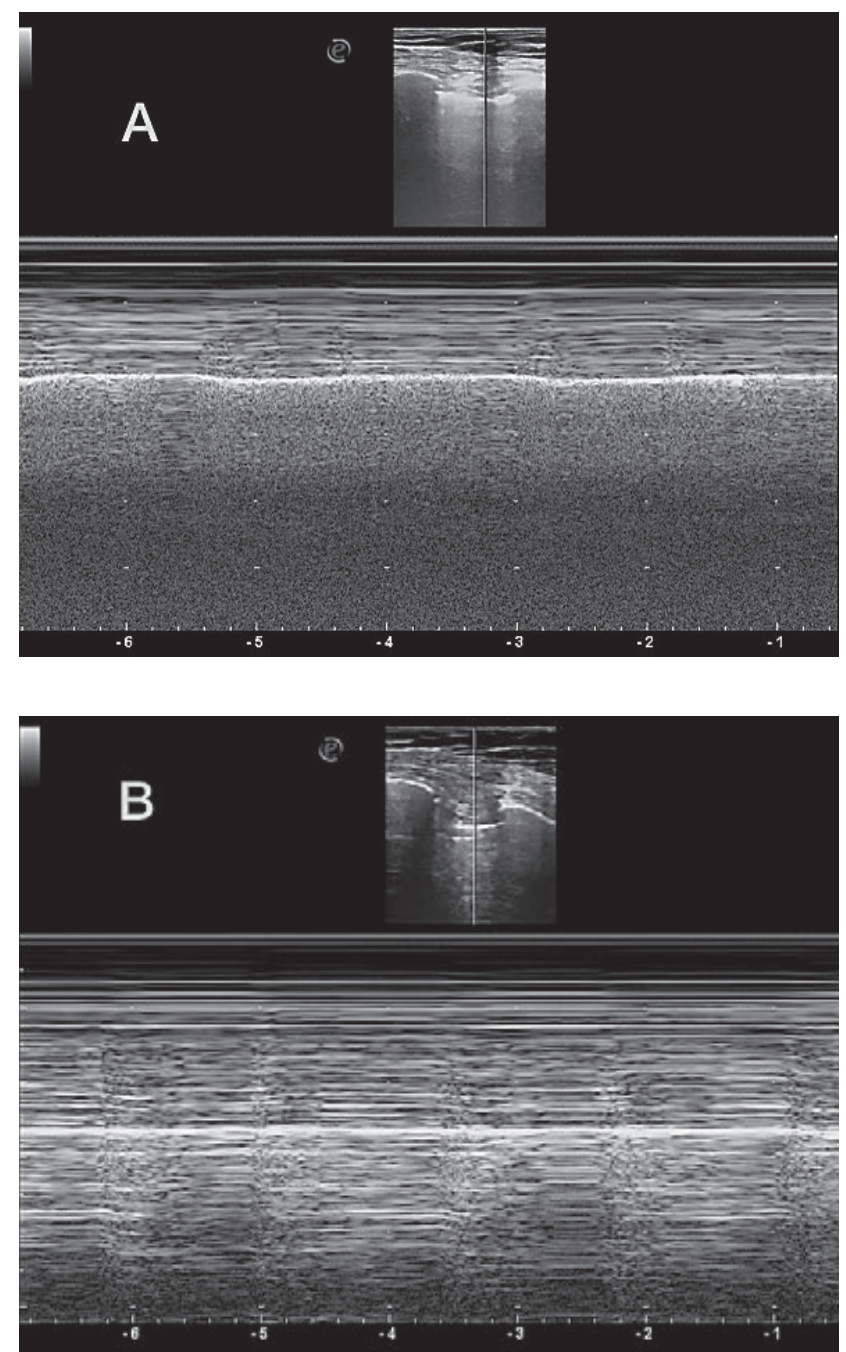

Figura 7. Planos ecocardiográficos básicos. A) Plano paraesternal eje largo; B) Plano paraesternal eje corto; C) Plano apical cuatro cámaras y D) Plano subxifoideo. Leyenda: VI: ventrículo izquierdo; VD: ventrículo derecho; Al: aurícula izquierda; $A D$ : aurícula derecha.

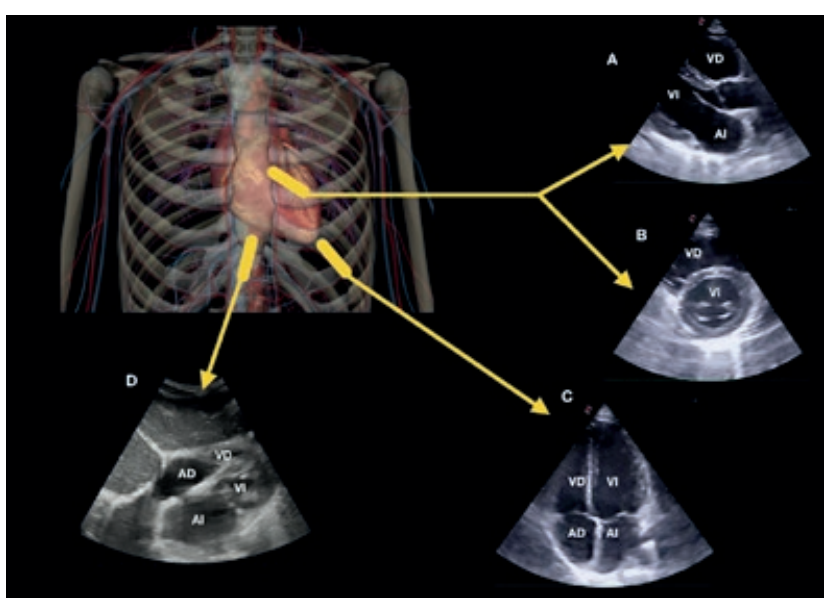




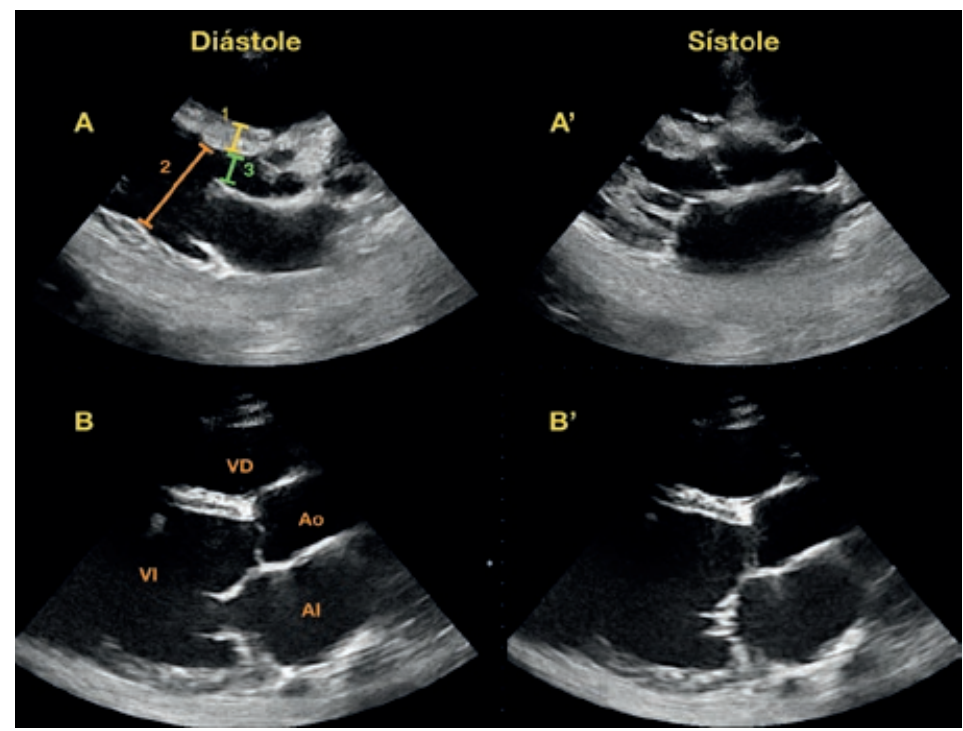

Figura 8. Claves para la estimación de la función sistólica del VI. En la parte superior de la imagen (A) se muestra el plano paraesternal longitudinal de un individuo sano con función sistólica conservada y en la parte inferior (B), el mismo plano en un enfermo con miocardiopatía dilatada y disfunción sistólica severamente deprimida.

Obsérvese en el individuo sano (A) el engrosamiento del septo interventricular en sístole con respecto a la diástole (1), el diámetro del VI y su acortamiento en sístole en comparación con la diástole (2) y como la valva anterior de la válvula mitral se aproxima al septo interventricular (punto e) en la diástole (3).

En cambio, en el enfermo con disfunción sistólica severa del VI (B) apenas se objetiva engrosamiento de la pared del septo interventricular, la valva anterior de la válvula mitral apenas se aproxima al septo interventricular durante la diástole y como el tamaño del VI está aumentado y apenas existe disminución del mismo en sístole.

Leyenda: VD: ventrículo derecho; VI: ventrículo izquierdo; Al: aurícula izquierda; Ao: aorta.

Aproximadamente en un 50\% de los pacientes con insuficiencia cardiaca la FEVI es normal. Muchos de estos enfermos tienen disfunción diastólica que puede sospecharse si detectamos hipertrofia ventricular izquierda y/o dilatación de la aurícula izquierda en ausencia de valvulopatía mitral (Figura 9).

La disfunción diastólica puede estimarse de forma relativamente sencilla valorando el flujo transmitral con el doppler pulsado (figura 10).

Por último, con la ecocardiografía clínica podemos hacer una estimación cualitativa de lesiones valvulares que pueden asociarse a insuficiencia cardiaca izquierda (estenosis aórtica, estenosis o insuficiencia mitral)(Figura 11).

La presencia de líneas B y/o derrame pleural bilateral en la ecografía pulmonar asociada a la detección de lesiones cardiacas significativas en el ecocardiograma (disfunción sistólica o diastólica ventricular izquierda, valvulopatía aórtica o mitral) permite establecer el diagnóstico de insuficiencia cardiaca con muy alta precisión (exactitud diagnóstica superior al $90 \%)^{2}$, por lo que se puede obviar la determinación de péptidos natriuréticos.
Figura 9. Hipertrofia ventricular izquierda severa en paciente con hipertensión arterial mal controlada (plano paraesternal eje largo). Obsérvese el grosor del septo interventricular (flecha). Leyenda: VD: ventrículo derecho; VI: ventrículo izquierdo; Al: aurícula izquierda; Ao: aorta.

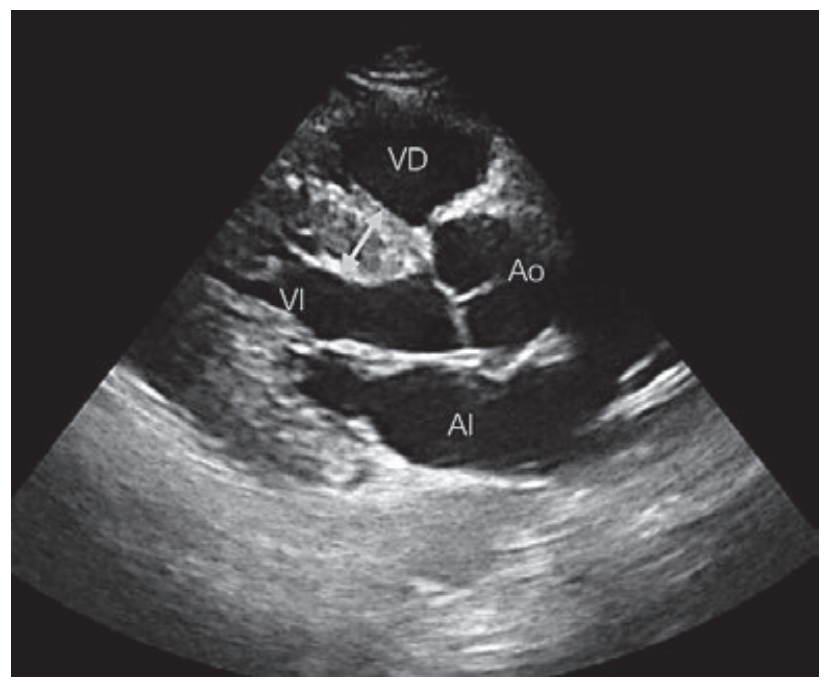

Figura 10. Flujo transmitral en paciente con miocardiopatía hipertensiva. Se aprecia una alteración de la relajación con velocidad de onda $E<$ onda $A$.

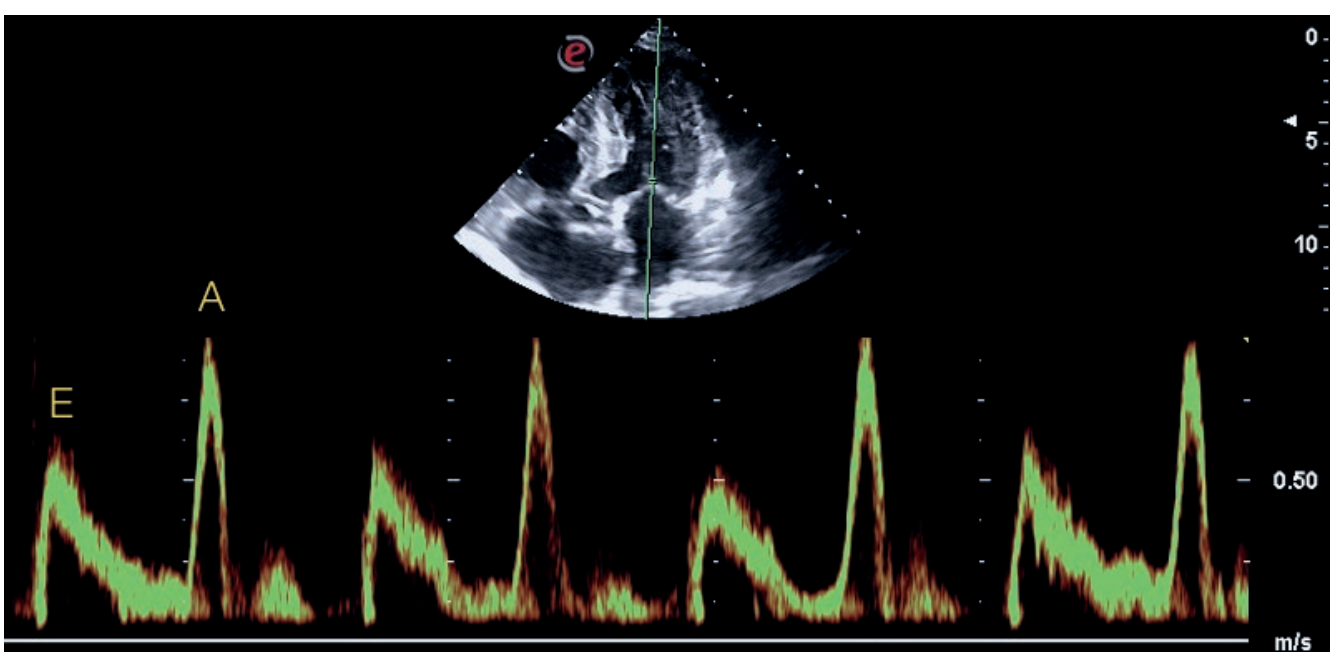


4. 2. 2. 2 Estimación de la presión venosa central (volemia) Desde el plano subxifoideo, tanto con la sonda sectorial como con la convex, podemos visualizar la vena cava inferior (VCI) desembocando en la aurícula derecha. El diámetro y el grado de colapsabilidad en inspiración de la VCI se relaciona con bastante exactitud con la presión venosa central (Figura 12). Esto permite al clínico hacer una estimación de la volemia y, por otra parte, también se relaciona con el grado de hipertensión pulmonar ${ }^{10}$. La valoración de la VCI tiene una gran importancia para estimar la afectación de cavidades derechas en enfermos con cardiopatía izquierda y, como veremos más adelante, para valorar la posibilidad de taponamiento cardiaco y de embolia de pulmón.

\section{2. 2. 3 Taponamiento cardiaco}

La detección de derrame pericárdico es bastante sencilla mediante la ecocardiografía. Se observa muy bien en el plano subxifoideo y no es necesario una curva de aprendizaje prolongada para su diagnóstico con fiabilidad ${ }^{11-19}$. El derrame pericárdico es moderado cuando su espesor supera los $10 \mathrm{~mm}$ y severo cuando es mayor de $20 \mathrm{~mm}$ (figura 13).

Hay diversos signos ecográficos que de forma sencilla orientan al diagnóstico de taponamiento cardiaco: derrame pericárdico severo, corazón que bambolea dentro del saco pericárdico ("swinging heart"), colapso de cavidades derechas y calibre de la VCl aumentado y sin colapso espiratorio. No obstante, no hay que olvidar que el diagnóstico del taponamiento cardiaco es clínico y es obligada la presencia de hipotensión y taquicardia. Si observamos estos signos y detectamos derrame pericárdico, independientemente de su cuantía, deberemos valorar la pericardiocentesis ${ }^{20}$.

\section{2. 2. 4 Valoración ecográfica del paciente con sospecha de tromboembolismo de pulmón}

Los hallazgos ecocardiográficos no son lo suficientemente específicos para diagnosticar un tromboembolismo pulmonar (TEP). Por tanto, esta herramienta nunca podrá susti-
Figura 11. A) Estenosis aórtica. Plano paraesternal eje largo en el que se observa una importante calcificación de la válvula aórtica (flecha). B) Estenosis mitral. Plano apical cuatro cámaras. Obsérvese la válvula mitral muy calcificada (flecha) y la enorme dilatación de la aurícula izquierda. Leyenda: VD: ventrículo derecho; VI: ventrículo izquierdo; Al: aurícula izquierda; Ao: aorta.
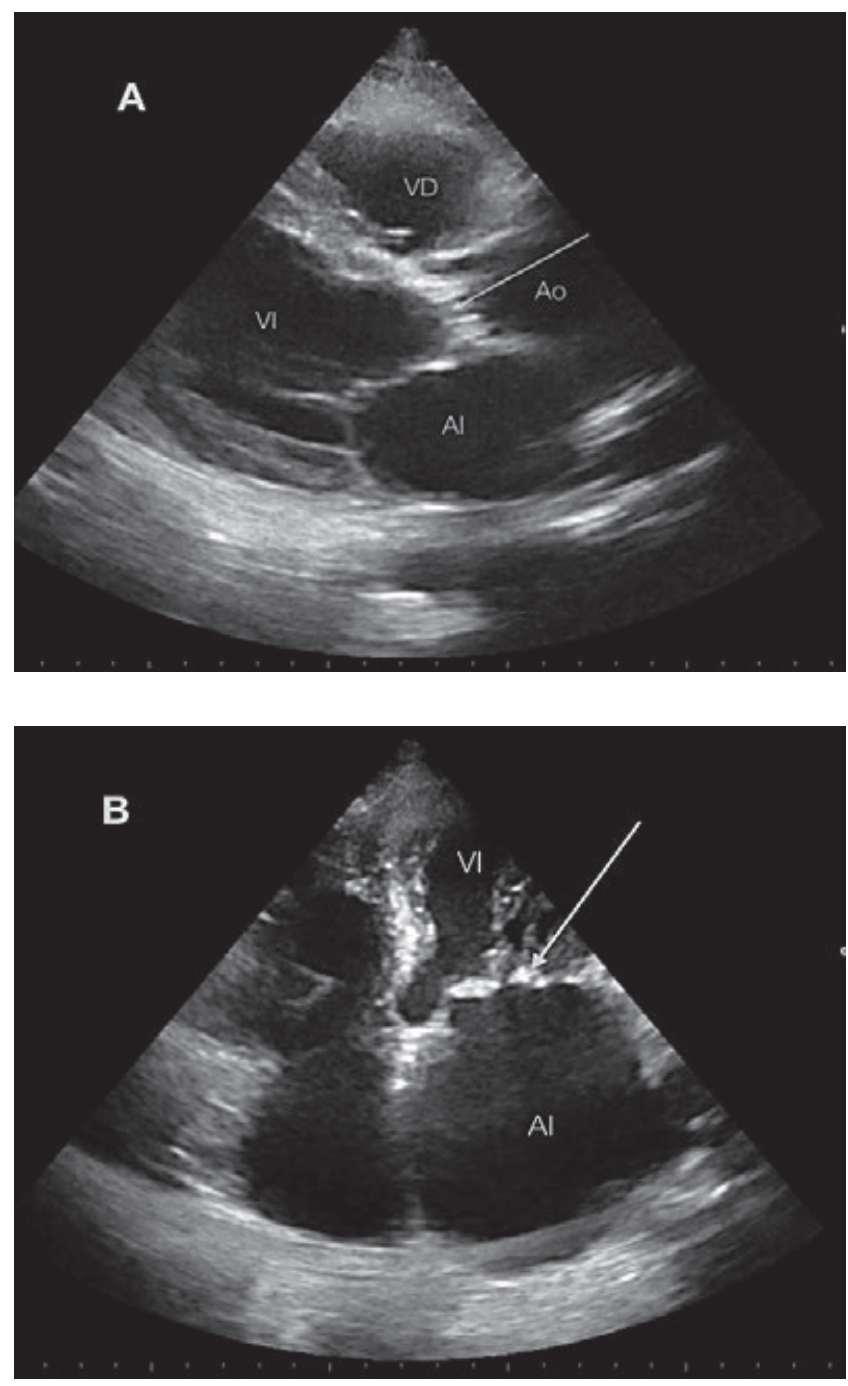

Figura 12. Plano longitudinal línea media en epigastrio en el que se observa la vena cava inferior (VCl) desembocando en la aurícula derecha (AD). A) Individuo con $\mathrm{VCl}$ filiforme con depleción de volumen y presión venosa central baja; B) Individuo con $\mathrm{VCl}$ de calibre normal y C) paciente con VCI muy dilatada en relación con cardiopatía e hipertensión pulmonar.

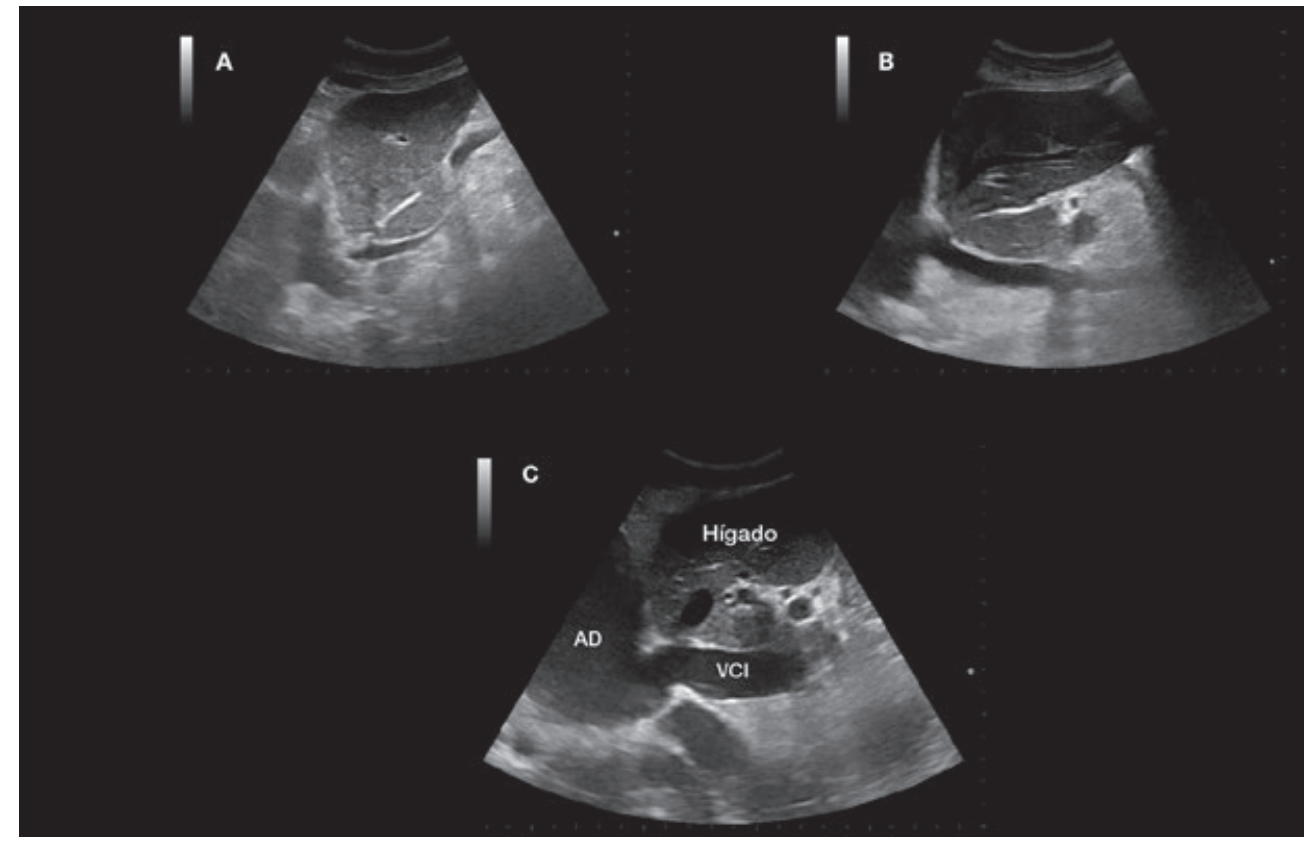


tuir al TAC o a la gammagrafía de pulmón para establecer el diagnóstico definitivo. Cuando la presión en el ventrículo derecho aumenta, como sucede de forma aguda en el TEP, sus paredes se arquean de forma que su tamaño iguala 0 incluso supera el del ventrículo izquierdo. Una norma general es que el cociente entre el diámetro del ventrículo derecho y el izquierdo (VD/VI) debe ser menor de 0,7 (plano apical cuatro cámaras), aunque en general se considera significativo un cociente superior a 1 (figura 14).

Por otra parte, un movimiento anormal del septo interventricular, que se dirija hacia el ventrículo izquierdo durante la diástole, es sugerente de aumento de la presión en ventrículo derecho. Un aumento del tamaño del ventrículo derecho junto con la presencia de un movimiento anormal del septo interventricular hacia el ventrículo izquierdo son signos muy sugerentes de hipertensión pulmonar.

En un paciente con sospecha embolia de pulmón e inestabilidad hemodinámica a veces no hay tiempo para confirmar el diagnóstico con otras pruebas (ej: TAC multicorte). En este contexto, si se detecta un ventrículo derecho dilatado e hipocontráctil, se debería plantear la trombolisis. Otros hallazgos ecocardiográficos que sugieren hipertensión pulmonar son la dilatación de la aurícula derecha, la presencia de insuficiencia tricúspide moderada o severa mediante Doppler color (figura 14) y la dilatación de la VCl. Es preciso recalcar que la presencia de estos signos no es específica del TEP y puede estar presente en pacientes con EPOC, SAHS, infarto del ventrículo derecho... .etc.

\section{3 Ecografía del sistema venoso profundo de las extremidades inferiores}

En un enfermo con sospecha de clínica de embolia de pulmón, también puede ser muy útil la ecografía del sistema venoso de extremidades inferiores ya que más del $50 \%$ de los pacientes con embolia de pulmón confirmada tienen TVP detectable en ecografía aunque no tengan clínica asociada a la misma. La detección de trombosis venosa profunda (TVP) confirma el diagnóstico de enfermedad tromboembólica.

\section{3. 1 Técnica}

Emplearemos una sonda lineal de alta frecuencia $(7-10 \mathrm{MHz})$. Realizaremos cortes a nivel inguinal y poplíteo. Si existe trombo en el interior de las venas estas no se pueden colapsar al comprimirlas e incluso es posible visualizar el trombo en su interior (figura 15).

\section{3. 2 El problema de la TVP distal}

La ecografía realizada por no radiólogos tiene unos valores de sensibilidad y especificidad superior al 95\% para la detección de TVP proximal. Sin embargo, entre un 20 y un 30\% de las TVP son distales; es decir, se localizan en las venas de la pantorrilla, distales al hueco poplíteo. La sensibilidad y especificidad de la ecografía para la detección de TVP distal es muy inferior a la de la TVP proximal.

Después del hueco poplíteo, la vena poplítea se divide en tres ramas: vena tibial anterior, vena tibial posterior y vena pero-
Figura 13. Plano paraesternal eje largo de paciente con derrame pericárdico severo (flechas). Obsérvese cómo el ventrículo derecho (VD) está parcialmente colapsado (VI: ventrículo izquierdo; Al: aurícula izquierda).

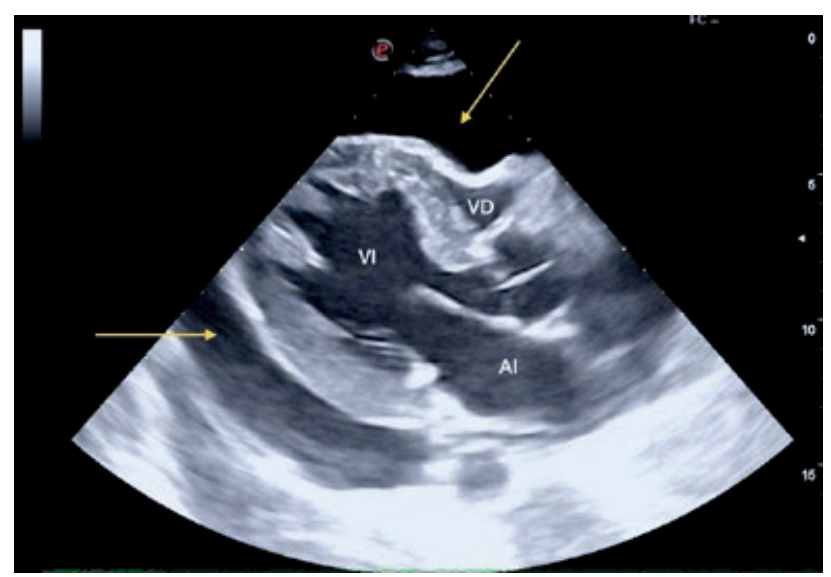

Figura 14. Ecocardiograma (plano apical cuatro cámaras) de un paciente con embolia de pulmón masiva. Obsérvese la gran dilatación de cavidades derechas y la insuficiencia tricuspídea (flecha).
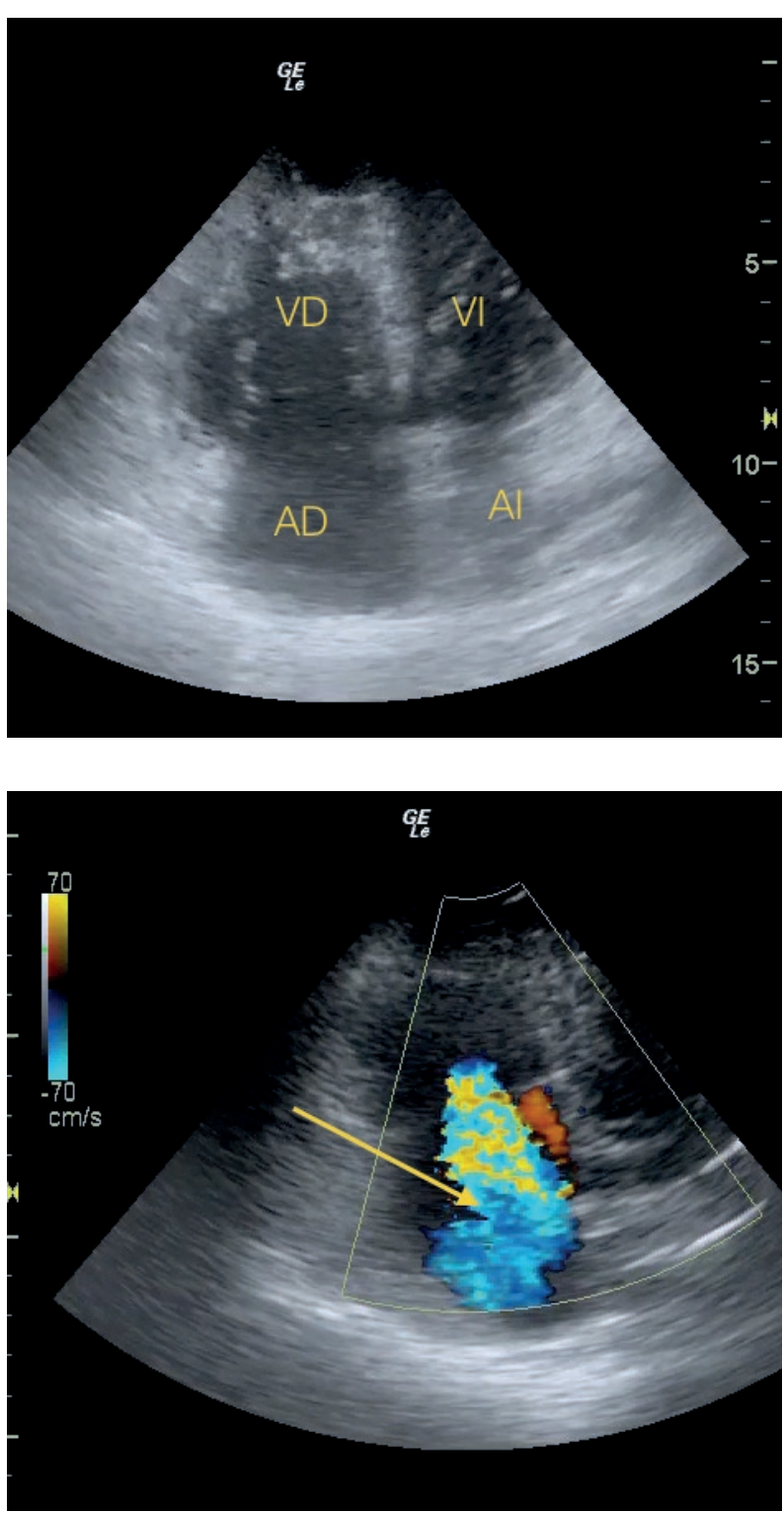
néa. A su vez poco después estas tres venas se bifurcan. Se trata de venas de pequeño grosor, difíciles de visualizar a lo largo de su trayecto. La sensibilidad máxima que se consigue para la detección de TVP distal en este territorio es del $70 \%$. Además, el tiempo que se tarda en explorar el sistema venoso profundo distal es considerable (seis territorios venosos en cada pierna).

El riesgo de que un paciente con TVP distal aislada se complique con una embolia de pulmón es muy bajo y no está suficientemente aclarado cuál es la mejor actitud terapéutica. Sin tratamiento específico sólo un 20\% progresan de las TVP distales progresan a territorio proximal. Tampoco hay evidencia de que el tratamiento de la TVP distal disminuya tanto la tasa de progresión como la de embolia de pulmón.

\section{Evidencias científicas de la utilidad de la ecografía clínica en el paciente con disnea}

Son varios los estudios clínicos publicados en revistas médicas indexadas en los que se demuestra la utilidad de la combinación de ecografía pulmonar, cardiaca y del sistema venoso de las extremidades inferiores en la valoración del paciente con disnea ${ }^{1-3,5,21-24}$.

En la mayoría de estos estudios se utilizan protocolos en los que se asocia la ecografía pulmonar a la ecocardiografía clínica, aunque en otros se añade además la ecografía de las extremidades inferiores 22, 25, 26 .

Las conclusiones más importantes de los mismos son las siguientes:
La ecografía clínica (pulmonar más cardiaca) permite establecer el diagnóstico de la etiología de la disnea con una precisión del 88-90\%, mientras que con la valoración clínica tradicional incluyendo pruebas complementarias (ECG, Rx tórax y analítica con BNP) la precisión es del 64-80\% ${ }^{5,25}$.

La presencia de líneas B bilaterales y en campos anteriores en la ecografía pulmonar permite establecer el diagnóstico de insuficiencia cardiaca aguda con una sensibilidad y especificidad de un $94 \%$ y de un $92,4 \%$, respectivamente ${ }^{1}$.

La ecografía clínica es especialmente útil para el diagnóstico de derrame pericárdico, derrame pleural paraneumónico, empiema y disfunción sistólica del ventrículo izquierdo, con una precisión diagnóstica que se aproxima al 100\% 25. La precisión diagnóstica para estas entidades con la valoración clínica tradicional es muy baja.

La precisión diagnóstica de la ecografía clínica para el diagnóstico de neumonía es del 86\% y para el EPOC 0 asma del 95\% ${ }^{5}$. La ecografía pulmonar es más sensible (> 95\%) y específica (95\%) que la determinación de péptidos natriuréticos (sensibilidad 75\%-85\% y especificidad 60\%-80\%) para el diagnóstico de insuficiencia cardiaca aguda 3, 21, 24, 27

La ecografía clínica puede realizarse antes de que el paciente con disnea llegue al hospital (medio extrahospitalario) y, por tanto, orientar el diagnóstico e iniciar el tratamiento específico con una alta fiabilidad ${ }^{27}$

La ecografía cardiaca, pulmonar y del sistema venoso profundo de extremidades inferiores permite establecer el diagnóstico de embolia de pulmón con una sensibilidad del 90\% y una especificidad del $86 \% 26$

Figura 15. Ecografía por compresión simplificada del

sistema venoso profundo proximal. Exploración a nivel inguinal $(A)$ en el que se observa

la vena femoral común (VFC) y las arterias femorales superficial (AFS) y profunda (AFP) y en hueco poplíteo (B) en el que se aprecia la vena poplítea (VP) y la arteria poplítea (AP). En condiciones normales, con una ligera presión se colapsan fácilmente las venas.

Sin embargo, si estas tienen un trombo en su interior no se colapsan. Además, en muchas ocasiones es posible visualizar el trombo en su interior, tal y como se aprecia en la parte inferior de la imagen. Las flechas señalan el trombo en la VFC y en la VP.

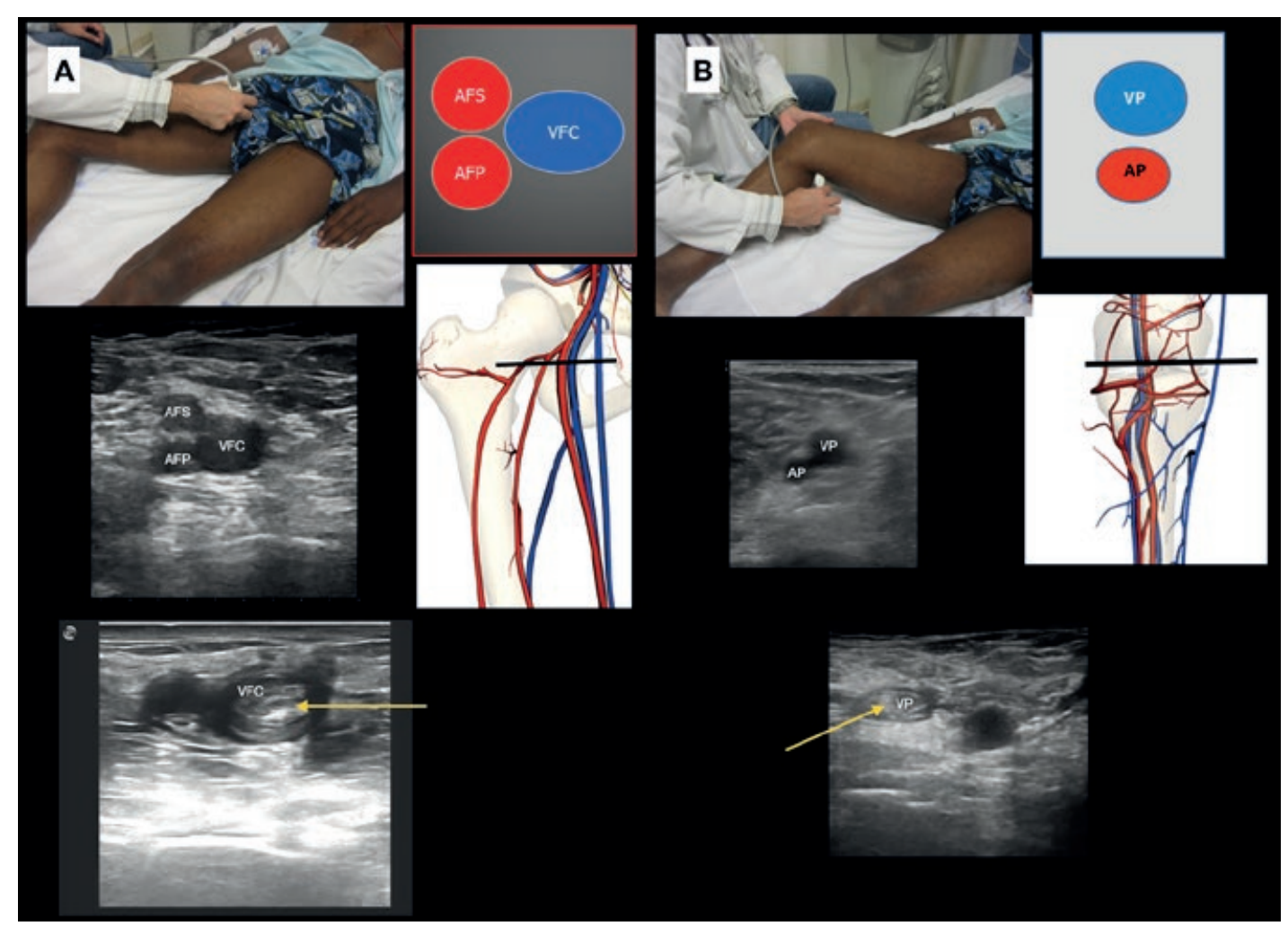




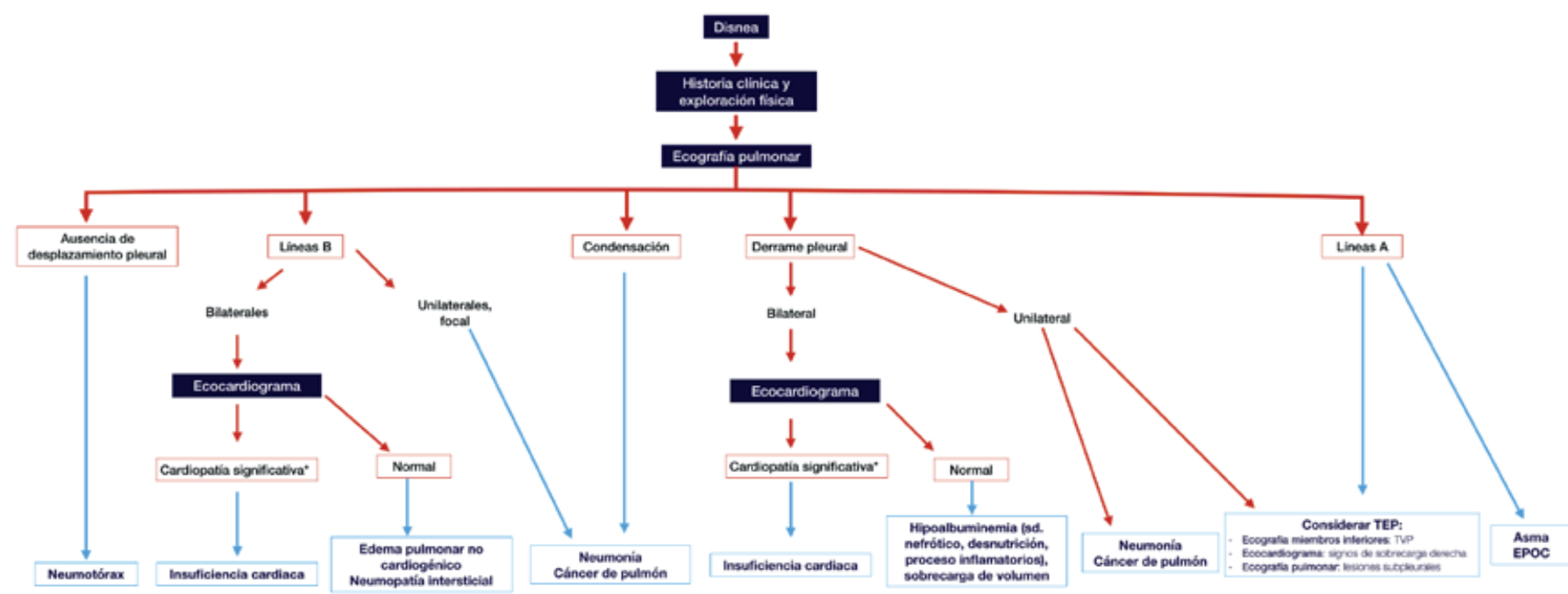

En más del 40\% de los pacientes con disnea de etiología incierta, la ecografía clínica proporciona datos sustanciales que condicionan modificaciones en su manejo y tratamiento².

Una exploración ecográfica pulmonar y cardiaca puede hacerse en menos de 15 minutos 2,5

En la figura 16 presentamos un algoritmo diagnóstico del paciente con disnea basado en la utilización de la ecografía clínica como complemento de la historia clínica y exploración física.

\section{Bibliografía}

1. Al Deeb M, Barbic S, Featherstone R, Dankoff J, Barbic D. Point-of-care ultrasonography for the diagnosis of acute cardiogenic pulmonary edema in patients presenting with acute dyspnea: a systematic review and meta-analysis. Acad Emerg Med 2014; 21: 843-852.

2. Russell FM, Ehrman RR, Cosby $K$ et al. Diagnosing acute heart failure in patients with undifferentiated dyspnea: a lung and cardiac ultrasound (LuCUS) protocol. Acad Emerg Med 2015; 22: 182-191.

3. Rempell JS, Noble VE. Using lung ultrasound to differentiate patients in acute dyspnea in the prehospital emergency setting. Crit Care 2011; 15: 161.

4. McMurray JJ, Adamopoulos S, Anker SD et al. ESC Guidelines for the diagnosis and treatment of acute and chronic heart failure 2012: The Task Force for the Diagnosis and Treatment of Acute and Chronic Heart Failure 2012 of the European Society of Cardiology. Developed in collaboration with the Heart Failure Association (HFA) of the ESC. Eur Heart J 2012; 33: 1787-1847.

5. Gallard E, Redonnet JP, Bourcier JE et al. Diagnostic performance of cardiopulmonary ultrasound performed by the emergency physician in the management of acute dyspnea. Am J Emerg Med 2015; 33: 352-358.

6. Nazerian P, Volpicelli G, Vanni S et al. Accuracy of lung ultrasound for the diagnosis of consolidations when compared to chest computed tomography. Am J Emerg Med 2015; 33: 620-625.

7. Raja AS, Jacobus $\mathrm{CH}$. How accurate is ultrasonography for excluding pneumothorax? Ann Emerg Med 2013; 61: 207-208.

8. Xirouchaki N, Magkanas E, Vaporidi K et al. Lung ultrasound in critically ill patients: comparison with bedside chest radiography. Intensive Care Med 2011; 37: 14881493.

9. Nazerian P, Vanni S, Zanobetti M et al. Diagnostic accuracy of emergency Doppler echocardiography for identification of acute left ventricular heart failure in patients with acute dyspnea: comparison with Boston criteria and $\mathrm{N}$-terminal prohormone brain natriuretic peptide. Acad Emerg Med 2010; 17: 18-26.

10. Yamanoglu A, Celebi Yamanoglu NG, Parlak I et al. The role of inferior vena cava diameter in the differential diagnosis of dyspneic patients; best sonographic measurement method. Am J Emerg Med 2015; 33: 396-401.

11. Andersen GN, Viset A, Mjolstad OC, Salvesen O, Dalen H, Haugen BO. Feasibility and accuracy of point-of-care pocket-size ultrasonography performed by medical students. BMC Med Educ 2014; 14: 156.
12. See KC, Ong V, Ng J, Tan RA, Phua J. Basic critical care echocardiography by pulmonary fellows: learning trajectory and prognostic impact using a minimally resourced training model*. Crit Care Med 2014; 42: 2169-2177.

13. Frederiksen CA, Juhl-Olsen $P$, Andersen NH, Sloth E. Assessment of cardiac pathology by point-of-care ultrasonography performed by a novice examiner is comparable to the gold standard. Scand J Trauma Resusc Emerg Med 2013; $21: 87$.

14. Liebo MJ, Israel RL, Lillie EO, Smith MR, Rubenson DS, Topol EJ. Is pocket mobile echocardiography the next-generation stethoscope? A cross-sectional comparison of rapidly acquired images with standard transthoracic echocardiography. Ann Intern Med 2011; 155: 33-38.

15. Andersen GN, Haugen BO, Graven T, Salvesen O, Mjolstad OC, Dalen H. Feasibility and reliability of point-of-care pocket-sized echocardiography. Eur J Echocardiogr 2011; 12: 665-670.

16. Vignon P, Mucke F, Bellec F et al. Basic critical care echocardiography: validation of a curriculum dedicated to noncardiologist residents. Crit Care Med 2011; 39, 636642.

17. Khasawneh FA, Smalligan RD. Focused transthoracic echocardiography. Postgrad Med 2010; 122: 230-237.

18. Lucas BP, Candotti C, Margeta B et al. Diagnostic accuracy of hospitalist-performed hand-carried ultrasound echocardiography after a brief training program. J Hosp Med 2009; 4: 340-349.

19. Vignon P, Dugard A, Abraham J et al. Focused training for goal-oriented hand-held echocardiography performed by noncardiologist residents in the intensive care unit. Intensive Care Med 2007; 33: 1795-1799.

20. Tayal VS, Kline JA. Emergency echocardiography to detect pericardial effusion in patients in PEA and near-PEA states. Resuscitation 2003; 59: 315-318.

21. Pivetta $E$, Goffi A, Lupia $E$ et al. Lung Ultrasound-Implemented Diagnosis of Acute Decompensated Heart Failure in the ED: A SIMEU Multicenter Study. Chest 2015; 148: 202-210.

22. Laursen CB, Sloth E, Lambrechtsen J et al. Focused sonography of the heart, lungs, and deep veins identifies missed life-threatening conditions in admitted patients with acute respiratory symptoms. Chest 2013; 144: 1868-1875.

23. Cibinel GA, Casoli G, Elia F et al. Diagnostic accuracy and reproducibility of pleural and lung ultrasound in discriminating cardiogenic causes of acute dyspnea in the emergency department. Intern Emerg Med 2012; 7: 65-70.

24. Anderson KL, Jenq KY, Fields JM, Panebianco NL, Dean AJ. Diagnosing heart failure among acutely dyspneic patients with cardiac, inferior vena cava, and lung ultrasonography. Am J Emerg Med 2013; 31: 1208-1214.

25. Laursen CB, Sloth E, Lassen AT et al. Point-of-care ultrasonography in patients admitted with respiratory symptoms: a single-blind, randomised controlled trial. Lancet Respir Med 2014; 2: 638-646.

26. Nazerian P, Vanni S, Volpicelli G et al. Accuracy of point-of-care multiorgan ultrasonography for the diagnosis of pulmonary embolism. Chest 2014; 145: 950-957.

27. Prosen G, Klemen P, Štrnad M, Grmec S. Combination of lung ultrasound (a comet-tail sign) and $\mathrm{N}$-terminal pro-brain natriuretic peptide in differentiating acute heart failure from chronic obstructive pulmonary disease and asthma as cause of acute dyspnea in prehospital emergency setting. Crit Care 2011; 15: R114. 